\title{
Large Scale Transmit Diversity in Q/V Band Feeder Link with Multiple Gateways
}

\author{
Ahmad Gharanjik*, Bhavani Shankar Mysore Rama Rao*, Pantelis-Daniel Arapoglou ${ }^{\dagger}$ and Björn Ottersten* \\ *Interdisciplinary Centre for Security, Reliability and Trust (SnT), University of Luxembourg \\ Email: \{ahmad.gharanjik, bhavani.shankar,bjorn.ottersten\}@uni.lu \\ $\dagger^{\dagger}$ ESA/ ESTEC, Noordwijk, Netherlands \\ Email: pantelis-daniel.arapoglou@esa.int
}

\begin{abstract}
Exploiting transmit diversity amid a high number of multiple gateways $(\mathrm{GW})$ is a new research challenge in $\mathrm{Q} / \mathrm{V}$ band satellite communication providing data rates of hundreds of Gbit/s. In this paper, we propose a practical switching strategy in a scenario with $N+P$ GWs ( $N$ active and $P$ redundant $\mathbf{G W s}$ ) towards achieving GW transmit diversity. Differently from other works, the treatment in this paper is analytical and explores two key factors: outage performance and switching rate in detail. Further, the interplay between the number of redundant and active GWs on the availability is illustrated highlighting the contribution of the work towards system sizing.

Index Terms-Gateway Diversity, active and redundant gateways, Q/V Band, Satellite Communication, Feeder Link.
\end{abstract}

\section{INTRODUCTION}

Demands on broadband data services are increasing dramatically every year. Although, satellite solutions have the advantage of covering these demands over a wide geography, to stay competitive with terrestrial solutions, it is necessary to push the limits of the capacity. Current satellite systems have capacity of about $70-100$ Gbps and it is estimated that next generation satellites will require capacity of one Terabit/s (1000 Gbps) by 2020 [1]. A key challenge to achieve a Terabit/s broadband satellite communication (SatCom) system is the limited spectrum of about $2 \mathrm{GHz}$ available in current $\mathrm{Ka}$ band. Following the traditional trend, this can be tackled by gradually shifting to a higher frequency band whenever the relevant technology is mature enough. Therefore, an attractive solution for resolving the issue is moving the feeder link from the Ka-band to the Q/V-band $(40 / 50 \mathrm{GHz})$ where larger bandwidth, up to $5 \mathrm{GHz}$, is available [1]-[3]. Further, it can free up the whole Ka-band spectrum for the user link. Moreover, it allows locating the gateways (GW) within the service area minimizing the interference between the feeder link and user link [3].

However, moving the feeder link to $\mathrm{Q} / \mathrm{V}$ band imposes considerable strain on the link-budget, predominantly due to heavy rain attenuation which is of the order of $15-20 \mathrm{~dB}$ [2]. The typical Fade Mitigation Technique (FMT) is the uplink power control. However, it can compensate only a few $\mathrm{dBs}$, thereby motivating the use of multiple GWs for transmit diversity to achieve the required availability in excess of $99.9 \%$ on the feeder link.

When the number of GWs is limited, the traditional $1+1$ scheme, where each GW is supported by a redundant GW can be an acceptable solution for GW transmit diversity. On the other hand, for high capacity satellite systems where tens of GWs are envisaged, it is not efficient to use traditional techniques. A system employing multiple GWs is the recently launched high throughput Ka-sat which has 82 spot beams and served by $10 \mathrm{GWs}$. Therefore designing smarter techniques for diversity becomes essential.

An interesting GW transmit diversity technique is $N+P$ diversity scheme, which was studied in [1], [4] and [5]. In this scheme, there are $\mathrm{N}$ active GWs and $\mathrm{P}$ redundant or idle GWs. When one of the active GWs is in outage, switching occurs and traffic of the active $\mathrm{GW}$ is rerouted to one of the idle GWs. Some works have been published exploring this scheme, for example [1], [4]-[7], but most of them take a high-level approach for system design without a rigorous mathematical analysis. To the best of our knowledge, the only work that has analysed GW diversity mathematically is [1] where authors derive the availability in a $N+P$ scenario. However, the authors in [1] do not describe if and how such an availability could be achieved. Further, the switching rate, which is an important system parameter is not studied in [1]. A high switching rate can lead to severe overheads and instability thereby warranting its further analysis. In this paper, we present a simple practical switching scheme well suited for the $N+P$ gateway scenario. Further, we derive closed form expressions for average outage performance and switching probability. These expressions provide insights on the tradeoffs between outage, switching rate and spectral efficiency while serving as tools for system sizing.

The remainder of this paper is organised as follows. Section II introduces the system and channel models for $N+P$ diversity scheme. In Section III, performance of the proposed scheme is evaluated analytically in terms of outage and switching probability. Numerical results are presented in Section IV. Concluding remarks are provided in Section V.

\section{Channel Model and GW Switching Strategy}

\section{A. Channel Model}

Consider a satellite feeder link with $N$ active GWs and $P$ idle GWs denoted by $G W_{a, n}(n=1, \ldots, N)$ and $G W_{b, k}(k=$ $1, \ldots, P)$ respectively. We assume that all GWs are connected to a Network Control Centre (NCC) node which has access to the channel state information of all GWs. The channels 
between the gateway $G W_{x, i},(x, i) \in\{(a, n),(b, k)\}$ and the satellite at $t=m T$ is denoted by $h_{x, i}[m]=\left|h_{x, i}[m]\right| e^{j \beta_{x, i}}$ where $\beta_{x, i}$ is the phase component. The channel amplitudes, $\left|h_{x, i}[m]\right|$, can be estimated at each GW using a beacon signal received from the satellite. Therefore, the corresponding Signal to Noise Ratio (SNR) for the active and idle GWs at $t=m T$ can be obtained by $\gamma_{x, i}[m]=\left|h_{x, i}[m]\right|^{2} \gamma_{C S_{U L}}$ where $\gamma_{C S_{U L}}$ is the clear sky SNR for the feeder uplink (from now on we will drop $m$ for simplicity).

In the $\mathrm{Q} / \mathrm{V}$ band - and in general in millimeter wave frequencies- the main impairment is the rain attenuation which is typically modelled using the lognormal distribution [9]. In our work, the other clear-sky effects are assumed to be compensated by a fixed fade margin or an uplink power control scheme. The rain attenuation and the channel gains are related as $A_{x, i}=-10 \log _{10}\left|h_{x, i}\right|^{2}$. The GWs are located far apart so that they can be assumed to experience independent rain attenuation. Also, for tractability of analysis, we assume identical rain attenuation statistics among the different GWs. This assumption is later shown to be mild when non identical distributions are considered in the numerical simulations. Under these assumptions, the corresponding rain attenuations $\left\{A_{a, n}\right\}_{n=1}^{N}$ and $\left\{A_{b, k}\right\}_{k=1}^{P}$ are i.i.d random variables with probability density function (PDF), $p_{A}($.$) and cumulative$ distribution function (CDF), $P_{A}($.$) . From [9], the lognormal$ $p_{A}($.$) takes the form$

$$
p_{A}(A)=\frac{1}{\sqrt{2 \pi} \sigma A} e^{-\frac{(\ln A-m)^{2}}{2 \sigma^{2}}} .
$$

Here, $m$ and $\sigma$ are the mean and standard deviation of $\ln A$ respectively. As a representative case, we use the parameters calculated for Luxembourg city using ITU-R Recommendation P.618 [9]. Due to independence of $\left\{A_{a, n}\right\}_{n=1}^{N}$ and $\left\{A_{b, k}\right\}_{k=1}^{P}$, we can assume that SNR of the active and idle GWs, $\left\{\gamma_{a, n}\right\}_{n=1}^{N}$ and $\left\{\gamma_{b, k}\right\}_{k=1}^{P}$, are i.i.d random variables with common PDF, $p_{\gamma}($.$) and \mathrm{CDF}, P_{\gamma}($.$) .$

\section{B. GW Switching Strategy}

Fig. 1 illustrates the switching strategy in detail. After collecting all SNRs, NCC sorts the active and idle GWs based on their SNR in decreasing order (this is same as sorting the GWs based on their rain attenuation in increasing order). The $n$th largest SNR of the active GWs and its corresponding GW are denoted by $\gamma_{(a, n)}$ and $G W_{(a, n)}$ respectively. For the idle GWs, the $k$ th largest SNR and its corresponding GW are depicted by $\gamma_{(b, k)}$ and $G W_{(b, k)}$ respectively. Therefore, we can write $\gamma_{(a, 1)} \geq \gamma_{(a, 2)} \ldots \geq \gamma_{(a, N)}$ and $\gamma_{(b, 1)} \geq \gamma_{(b, 2)} \ldots \geq \gamma_{(b, P)}$. According to the relation between the channel gain and rain attenuation, we have $\left|h_{(a, i)}\right|^{2}=10^{-A_{[a, i]}}$, where $A_{[a, i]}$ is the $i$ th smallest value of the rain attenuation. Hence, we can write $A_{[a, 1]} \leq A_{[a, 2]} \leq \ldots \leq A_{[a, N]}$ and $A_{[b, 1]} \leq A_{[b, 2]} \leq \ldots \leq$ $A_{[b, P]}$.

After the sorting step, NCC initiates switching between idle $G W_{(b, k)}$ and active $G W_{\left(a, k^{\prime}\right)}$, where $k=1, \ldots, P$ and $k^{\prime}=$ $N-k+1$. Thus, $P$ switching pairs will be formed such that the weakest active GW, $G W_{(a, N)}$, will have the best chance to

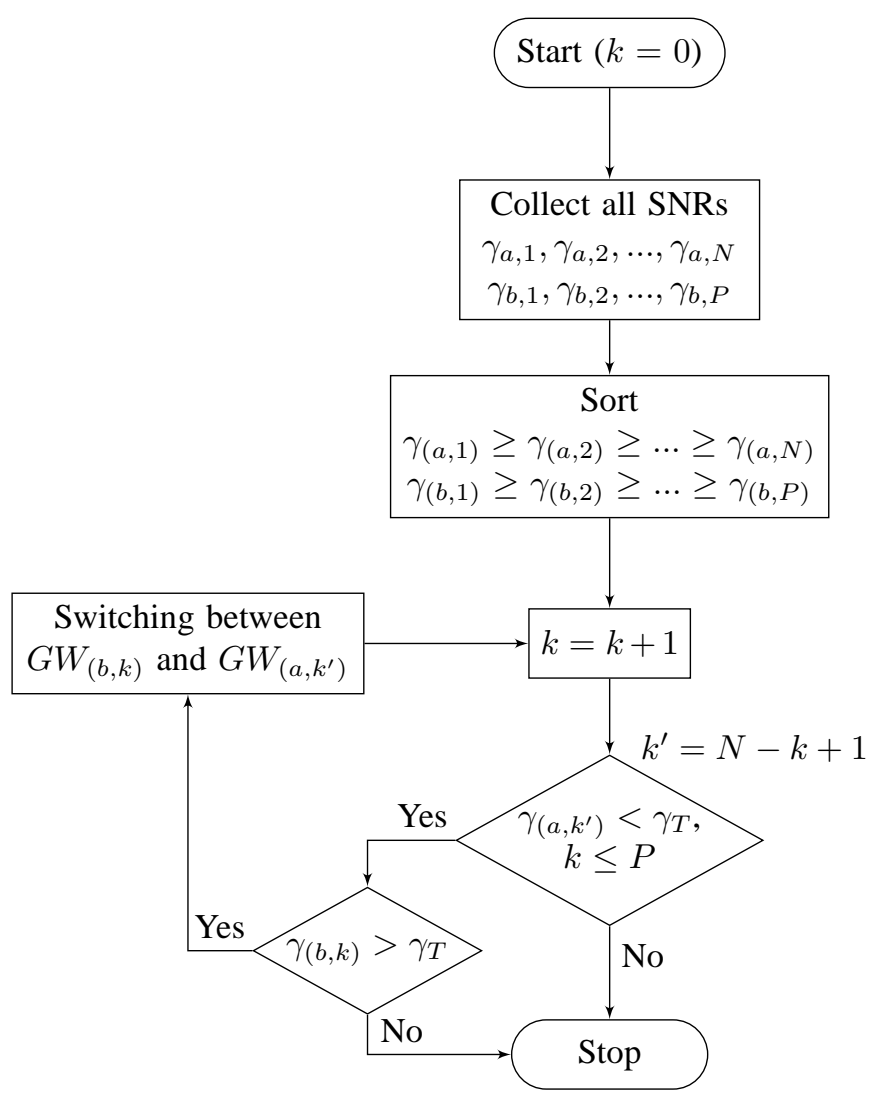

Fig. 1. Flowchart of $\mathrm{N}+\mathrm{P}$ Gateway Switching scheme

switch to the strongest idle GW, $G W_{(b, 1)}$. The switching will take place based on a scheme similar to modified switch and stay combining (MSSC) technique introduced for two GWs in [8]. In this MSSC based switching method, if $\gamma_{\left(a, k^{\prime}\right)}$ is lower than $\gamma_{T}$ and $\gamma_{(b, k)}$ is higher than $\gamma_{T}$, switching occurs between two GWs. Here, $\gamma_{T}$ is switching threshold and its selection will be detailed in the sequel.

If the switching takes place for the first pair, i.e. between $\gamma_{(b, 1)}$ and $\gamma_{(a, N)}$, the NCC will continue the switching process for the subsequent pairs upon investigating the necessity of switching for those as well. Note that in each time slot, the state of the GWs (active or idle) might change due to switching. However, this will not impact the ensuing statistical analysis since the SNR associated with different GWs have independent and identical distribution.

\section{PeRformance STUdy}

\section{A. Outage Probability}

In this subsection, we will study the performance of the proposed scheme in terms of average outage. We denote the outage threshold by $\gamma_{t h}$ and define the average outage probability of the system as

$$
\bar{P}_{\text {out }}\left(\gamma_{t h}\right)=\frac{1}{N}\left(\sum_{n=1}^{N-P} P_{\text {out }, n}^{N S}\left(\gamma_{t h}\right)+\sum_{k=1}^{P} P_{\text {out }, k}^{S}\left(\gamma_{t h}\right)\right) .
$$




$$
\bar{P}_{\text {out }}\left(\gamma_{t h}\right)=\frac{1}{N} \sum_{n=1}^{N-P}\left(1-P_{A_{[a, n]}}\left(\alpha_{t h}\right)\right)+\frac{1}{N} \sum_{k=1}^{P} \frac{\left(2-P_{A_{\left[a, k^{\prime}\right]}}\left(\alpha_{t h}\right)-P_{A_{[b, k]}}\left(\alpha_{t h}\right)\right)}{1 /\left(1-P_{A_{\left[a, k^{\prime}\right]}}\left(\alpha_{T}\right)\right)+1 /\left(1-P_{A_{[b, k]}}\left(\alpha_{T}\right)\right)} .
$$

Here $P_{o u t, n}^{N S}\left(\gamma_{t h}\right), 1 \leq n \leq N-P$, is the outage probability of each of the $N-P$ GWs that are not involved in the switching process. Further, $P_{\text {out }, k}^{S}\left(\gamma_{t h}\right)$ is the outage probability of each of the $P$ switching pairs.

We now evaluate $P_{\text {out }, k}^{S}\left(\gamma_{t h}\right)$ and $P_{o u t, n}^{N S}\left(\gamma_{t h}\right)$. Assuming $\gamma_{T} \geq \gamma_{t h}$, the outage probabilities of the switching pairs, $P_{o u t, k}^{S}\left(\gamma_{t h}\right)$, can be calculated using the results of [10] as,

$$
P_{o u t, k}^{S}\left(\gamma_{t h}\right)=\frac{P_{\gamma_{\left(a, k^{\prime}\right)}}\left(\gamma_{t h}\right)+P_{\gamma_{(b, k)}}\left(\gamma_{t h}\right)}{1 / P_{\gamma_{\left(a, k^{\prime}\right)}}\left(\gamma_{T}\right)+1 / P_{\gamma_{(b, k)}}\left(\gamma_{T}\right)},
$$

where $P_{\gamma_{(x, i)}}(z)=\operatorname{Pr}\left\{\gamma_{(x, i)} \leq z\right\}, x \in\{a, b\}$. The outage probability of the remaining $N-P$ active GWs, that are not involved in the switching process can be calculated as

$$
\begin{aligned}
P_{o u t, n}^{N S}\left(\gamma_{t h}\right) & =\operatorname{Pr}\left\{\gamma_{(a, n)} \leq \gamma_{t h}\right\}=\operatorname{Pr}\left\{A_{[a, n]}>\Gamma_{C S}-\Gamma_{t h}\right\} \\
& =1-P_{A_{[a, n]}}\left(\Gamma_{C S}-\Gamma_{t h}\right), 1 \leq n \leq N-P, \quad(5)
\end{aligned}
$$

where $\Gamma_{C S}=10 \log \gamma_{C S}, \Gamma_{t h}=10 \log \gamma_{t h}$ and $P_{A_{[a, n]}}($. is the CDF of $n$th order statistics. Similarly, we can find that $P_{\gamma_{(u, v)}}\left(\gamma_{t h}\right)=1-P_{A_{[u, v]}}\left(\alpha_{t h}\right)$ and $P_{\gamma_{(u, v)}}\left(\gamma_{T}\right)=1-$ $P_{A_{[u, v]}}\left(\alpha_{T}\right)$ where $u \in\{a, b\}, v \in\left\{k, k^{\prime}\right\}, \alpha_{t h}=\Gamma_{C S}-\Gamma_{t h}$, $\alpha_{T}=\Gamma_{C S}-\Gamma_{T}$ and $\Gamma_{T}=10 \log \gamma_{T}$. In the above expressions, $P_{A_{[a, v]}}$ and $P_{A_{[b, v]}}$ can be obtained from [11] as,

$$
\begin{gathered}
P_{A_{[a, v]}}(\alpha)=\sum_{t=v}^{N}\left(\begin{array}{c}
N \\
t
\end{array}\right)\left[P_{A}(\alpha)\right]^{t}\left(1-P_{A}(\alpha)\right)^{N-t}, \\
P_{A_{[b, v]}}(\alpha)=\sum_{t=v}^{P}\left(\begin{array}{c}
P \\
t
\end{array}\right)\left[P_{A}(\alpha)\right]^{t}\left(1-P_{A}(\alpha)\right)^{P-t} .
\end{gathered}
$$

It remains to evaluate $P_{A}(\alpha)$ towards obtaining (3). Since rain attenuation follows the log-normal distribution as in (1), $P_{A}(\alpha)$ can be obtained as

$$
\begin{aligned}
P_{A}(\alpha) & =\int_{0}^{\alpha} \frac{1}{\sqrt{2 \pi} \sigma A} e^{-\frac{(\ln A-m)^{2}}{2 \sigma^{2}}} d A \\
& =0.5+0.5 \operatorname{erf}\left(\frac{\ln \alpha-m}{\sqrt{2} \sigma}\right) .
\end{aligned}
$$

Finally, by substituting (4) and (5) in (3) we get an expression for average outage probability of the system given in (2), top of the next page.

Remark 1: Effect of switching threshold on outage: From (4), it is easy to show that the optimum switching threshold $\left(\gamma_{T}\right)$ in the sense of minimum outage probability is $\gamma_{T}=\gamma_{t h}$. In this case, (4) can be simplified as

$$
P_{o u t, k}^{S}\left(\gamma_{t h}\right)=P_{\gamma_{\left(a, k^{\prime}\right)}}\left(\gamma_{t h}\right) P_{\gamma_{(b, k)}}\left(\gamma_{t h}\right) .
$$

Remark 2: Effect of switching threshold on spectral efficiency: Clearly, when a higher threshold is chosen, the GW supports a spectrally more efficient transmission when active.
TABLE I

PROPAGATION AND LINK BUDGET ASSUMPTIONS

\begin{tabular}{c|c}
\hline Feeder Up-Link & Value \\
\hline Carrier frequency & $50 \mathrm{GHz}$ \\
Elevation angle & $32^{\circ}$ \\
Polarization & Circular \\
\hline EIRP $_{G W}$ including back-off & $76.5 \mathrm{dBW}$ \\
UL free space loss & $218.3 \mathrm{~dB}$ \\
$(\mathrm{G} / \mathrm{T})_{S a t}$ & $31.45 \mathrm{~dB}$ \\
$\gamma_{C S_{U L}}$ & $28.3 \mathrm{~dB}$ \\
\hline
\end{tabular}

This leads to an improved spectral efficiency for the whole system.

In Section IV, we will discuss more about the effect of choosing different $\gamma_{T}$.

\section{B. Switching Rate}

When a GW switching strategy is used, the switching rate is an important issue since a high switching rate results in high overhead and can make the system unstable. Therefore, in this subsection we analyze the switching rate of the $N+P$ diversity scheme. As explained in subsection II-B, switching will occur between $\gamma_{\left(a, k^{\prime}\right)}$ and $\gamma_{(b, k)}$ based on MSSC scheme. Hence, similar to the approach used in [8], it is possible to define a six state Markov chain model for each switching pair. The transitional probability matrix $\mathbf{P}$ of the Markov chain can be obtained as( for details kindly refer to [8]),

$$
\mathbf{P}=\left(\begin{array}{cccccc}
1-p_{a, k^{\prime}} & p_{k} & p_{a, k^{\prime}}-p_{k} & 0 & 0 & 0 \\
1-p_{a, k^{\prime}} & p_{k} & p_{a, k^{\prime}}-p_{k} & 0 & 0 & 0 \\
0 & 0 & 0 & 1-p_{b, k} & p_{k} & p_{b, k}-p_{k} \\
0 & 0 & 0 & 1-p_{b, k} & p_{k} & p_{b, k}-p_{k} \\
0 & 0 & 0 & 1-p_{b, k} & p_{k} & p_{b, k}-p_{k} \\
1-p_{a, k^{\prime}} & p_{k} & p_{a, k^{\prime}}-p_{k} & 0 & 0 & 0
\end{array}\right)
$$

where $p_{a, k^{\prime}}=P_{\gamma_{\left(a, k^{\prime}\right)}}\left(\gamma_{T}\right), p_{b, k}=P_{\gamma_{(b, k)}}\left(\gamma_{T}\right)$ and $p_{k}=$ $P_{\gamma_{\left(a, k^{\prime}\right)}}\left(\gamma_{T}\right) P_{\gamma_{(b, k)}}\left(\gamma_{T}\right)$. We define $\pi_{i, k}$ as the probability that $k$ th switching pair is in state $i$. By using the facts that $\vec{\pi}=$ $\vec{\pi} \mathbf{P}$ and $\sum_{i=1}^{6} \pi_{i, k}=1$, where $\vec{\pi}=\left[\pi_{1, k}, \pi_{2, k}, \ldots, \pi_{6, k}\right]$, switching probability of $k$ th pair can be calculated as

$$
\pi_{k}\left(\gamma_{T}\right)=\frac{2\left(p_{a, k^{\prime}}-p_{k}\right)\left(p_{b, k}-p_{k}\right)}{p_{a, k^{\prime}}+p_{b, k}-2 p_{k}},
$$

Now, we can define the average switching probability as

$$
\pi=\frac{1}{P} \sum_{k=1}^{P} \pi_{k}\left(\gamma_{T}\right)
$$

Now, the switching rate can be easily calculated as $\pi / T$ where $T$ is the interval between switching instants. 
TABLE II

DVB-S2 MODCOD SCHEME

\begin{tabular}{c|c}
\hline ModCod & $\Gamma_{T}[\mathrm{~dB}]$ \\
\hline \hline QPSK 1/4 & -2.72 \\
\hline QPSK 1/3 & -1.52 \\
\hline QPSK 1/2 & 0.73 \\
\hline QPSK 3/5 & 1.93 \\
\hline QPSK 2/3 & 2.83 \\
\hline QPSK 3/4 & 3.78 \\
\hline QPSK 5/6 & 4.83 \\
\hline 8PSK 3/5 & 5.33 \\
\hline 8PSK 2/3 & 6.43 \\
\hline 8PSK 3/4 & 7.63 \\
\hline 16APSK 2/3 & 9.95 \\
\hline 16APSK 3/4 & 11.20 \\
\hline 16APSK 4/5 & 12.05 \\
\hline 16APSK 5/6 & 12.60 \\
\hline 32APSK 3/4 & 14.58 \\
\hline 32APSK 4/5 & 15.08 \\
\hline 32APSK 5/6 & 16.18 \\
\hline
\end{tabular}

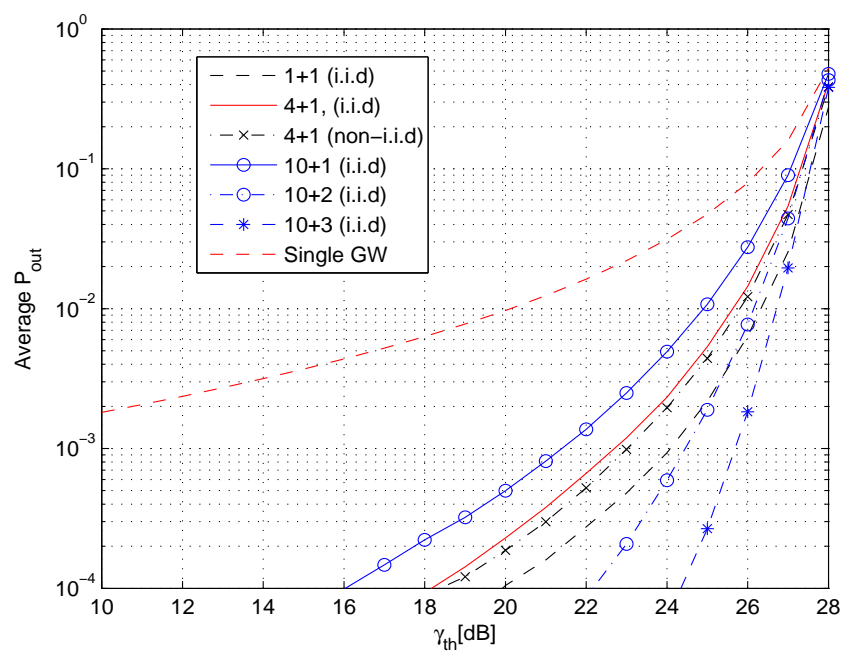

Fig. 2. Average outage probability for different $N+P$ configurations

\section{NUMERICAL Results AND Discussion}

Table I details the propagation parameters that were used as input to the empirical rain attenuation prediction model included in ITU-R Recommendation P.618 [9]. It also presents the forward feeder link budget that has been used in the numerical results.

Fig. 2 illustrates the feeder-link outage performance of the proposed scheme for different configurations when the optimum switching threshold is selected, $\gamma_{T}=\gamma_{t h}$. It is aimed at providing some insights about the effect of $N, P$ on performance and aid in system design. We can see that, with the number of idle GWs fixed $(P=1)$, the outage probability degrades gracefully increasing the number of active GWs. This means that if we assign only one idle GW for 10 active GWs,

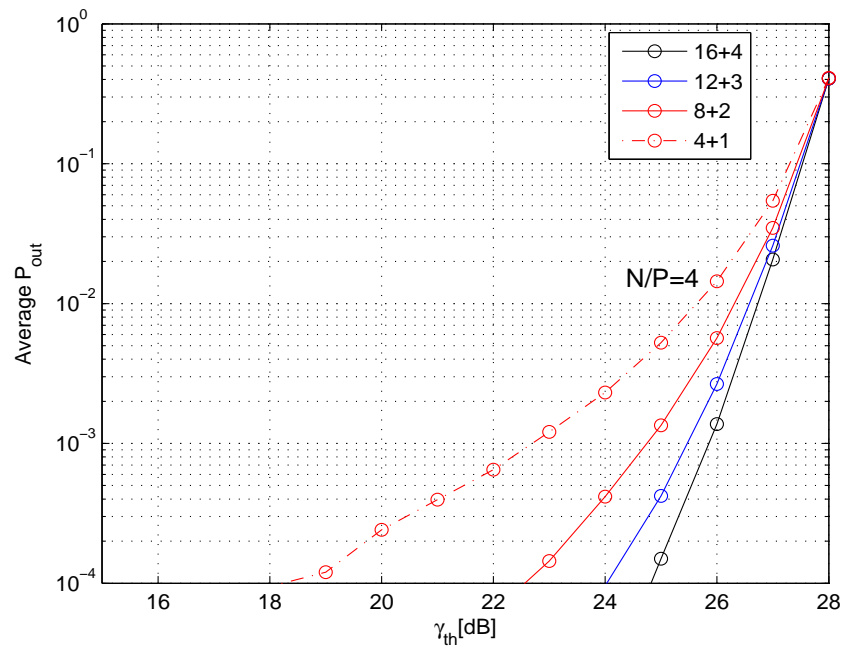

Fig. 3. Average outage probability for $\frac{N}{P}=4$ and different $N+P$ schemes

this scheme could still provide acceptable outage performance. Also, it can be seen that for a fixed number of active GWs $(N=10)$, if we increase the number of idle GWs for 1 to 2 outage probability decreases considerably.

In Fig. 2, we also plotted the outage probability for non i.i.d $4+1$ case. To study the performance of the system in a more realistic (non i.i.d) scenario, we located GWs in five European cities (Luxembourg, London, Amsterdam, Berlin and Athens) with different rainfall characteristics. We then compared the result with the i.i.d case. As can be see from Fig. 2, the i.i.d and non i.i.d case have very similar outage performance. Therefore, we can conclude that considering i.i.d rain attenuation is an acceptable assumption and will not considerably change the results of this study. So, we will focus on i.i.d case in the simulations. It is worth mentioning that all results except $4+1$ non i.i.d case are theoretical evaluations.

Fig. 3 presents the average outage probability of different configurations when $\frac{N}{P}=4$. It can be inferred from the figure that for a fixed ratio of $\frac{N}{P}$, if the number of GWs increases, the system will have a better outage performance. This means that, for example, if there are 8 active GWs and 2 idle GWs, $8+2$ architecture will result in better overall performance than two $4+1$ cluster.

Fig. 4 presents the average availability of the large scale GW diversity scheme ( $1-\bar{P}_{\text {out }}$ in percentage) versus unavailability of a single GW $\left(1-P_{A}\left(\alpha_{t h}\right)\right.$ in percentage). For the case of $4+1$ and $7+1$, it can be seen that if availability of each GW is $99 \%$, the average availability of the whole GW network will be around $99.97 \%$ and $99.96 \%$ respectively.

Fig. 5 shows the influence of different switching thresholds, $\gamma_{T}$, on the switching probability, average outage probability and the spectral efficiency of the system. In fact, $\gamma_{T}$ is chosen correspond to the minimum $\frac{E_{s}}{N_{0}}$ required to support a certain Modcod in DVB-S2 [12]. The thresholds and the associated Modcods are presented in Table II for completeness.

The trade-off between spectral efficiency and outage prob- 


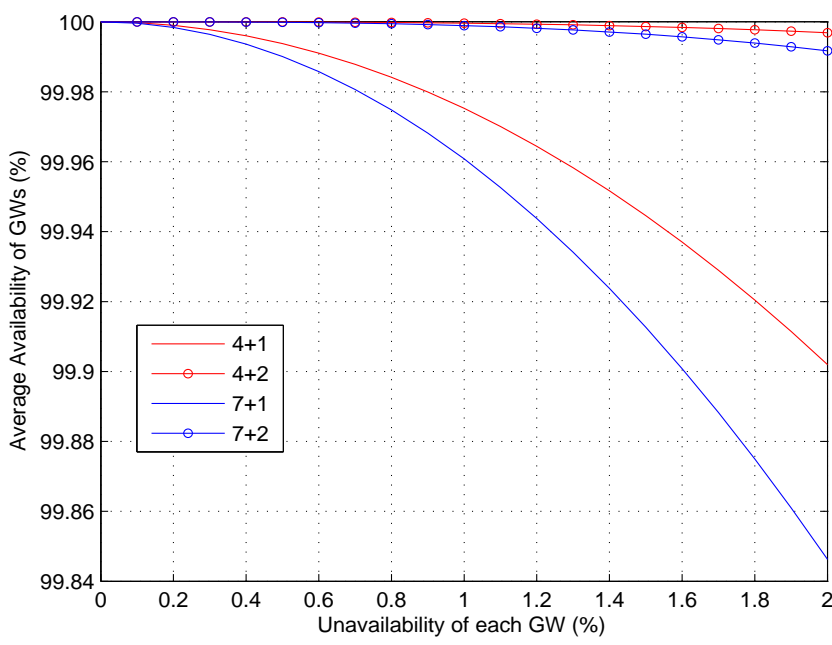

Fig. 4. Average availability of the GWs versus unavailability of a single GW

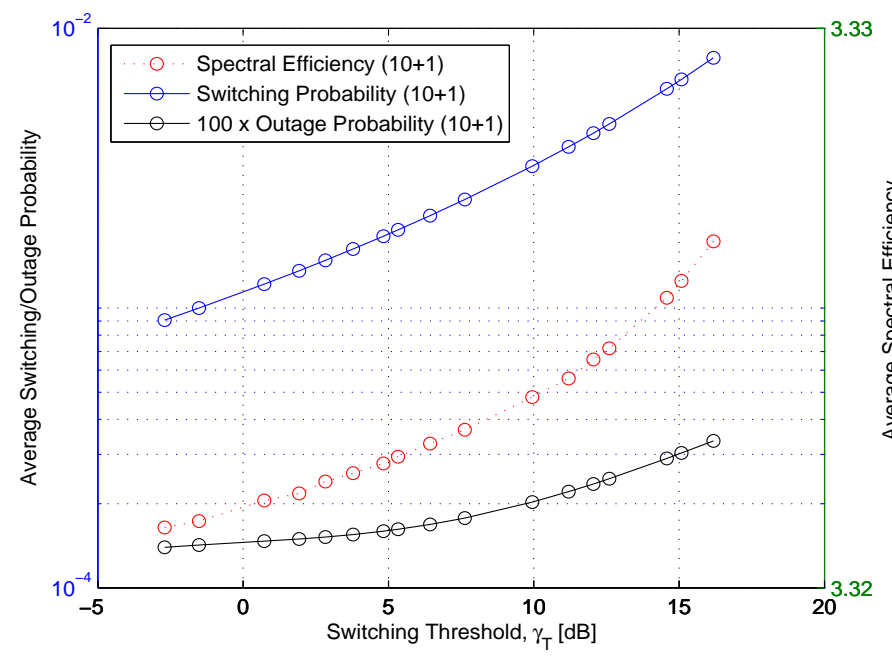

Fig. 5. Average Switching probability for different switching thresholds

ability caused by threshold selection has been detailed in remarks 1 and 2 of Section III. However, from Fig. 5, the improvements in spectral efficiency are negligible while the switching rate is sensitive to the threshold. This naturally leads to the choice of threshold resulting in an optimal outage/ switching rate for the considered scenario. As expected, by increasing $\gamma_{T}$, the switching probability of the system increases and so does the spectral efficiency.

\section{CONCLUSION}

In this paper, we devised a realistic switching scheme to exploit large scale diversity in multiple GW scenario necessary when moving the feeder link of a multibeam boradband satellite network to $\mathrm{Q} / \mathrm{V}$ band. Two novel aspects of the proposed scheme are the association of GWs into switching pairs based on ordered SNR and the use of robust MSSC strategy. Expressions for key performance indicators - outage and switching rate - have been derived providing insights into system sizing especially on the relative effect of the number of idle and active GWs. An interesting result is that bigger clusters yield better performance for a fixed ratio of idle and active GWs.

\section{ACKNOWLEDGMENT}

This work was supported by the National Research Fund, Luxembourg under AFR grant for Ph.D. project (Reference 5779106) on "Transmission and Reception Techniques for Smart Gateways in Next Generation Satellite Systems".

\section{REFERENCES}

[1] A. Kyrgiazos, B. Evans, P. Thompson and N. Jeannin, Gateway Diversity scheme for a Future Broadband Satellite System, 6th Advanced Satellite Multimedia Systems Conference (ASMS), Baiona, September, 2012.

[2] P. D. Arapoglou, M. R. B. Shankar, A. D. Panagopoulos and B. خे Ottersten, Gateway Diversity Strategies in $Q / V$ Band Feeder Links, 17th Ka and Broadband Communications Conference, Palermo, Italy, 3-5.October, 2011.

[3] O. Vidal, G. Verelst, J. Lacan, E. Alberty, J. Radzik, and M. Bousquet, Next Generation High Throughput Satellite System, IEEE First AESS European Conference on Satellite Telecommunications (ESTEL), vol., no., pp.1,7, 2-5 Oct. 2012.

$\stackrel{\Phi}{\Phi}[4]$ D. Mignolo, E. Re, A. Ginesi, A. B. Alamanac, P. Angeletti, and M. Harverson, Approaching terabit/s satellite: a system analysis, in 17th $\mathrm{Ka}$ and Broadband Communications Conference, 2011.

[5] P. Angeletti, R. De Gaudenzi and E. Re, Smart Gateway Diversity, Patent Description, European Space Agency, 2012.

[6] H. Skinnemoen, Gateway diversity in Ka-band systems, 4th Ka-band Utilization conference, Venice, Italy, 2-4 Nov 1998.

[7] N. Jeannin, L. Castanet, J. Radzik, M. Bousquet, B. Evans and P. Thompson, Assessment of the availability of Different Macro-Diversity Schemes for Q/V Band Feeder Links of Satcom Systems Targetting the $\mathrm{Tb} / \mathrm{s}$, in $17 \mathrm{th} \mathrm{Ka}$ band Broadband Communications, Navigation and Earth Observation Conference, Italy, Oct. 2011.

[8] A. Gharanjik, M. R. B. Shankar, P. D. Arapoglou and B. Ottersten, Gateway Switching in $Q / V$ Band Satellite Feeder Links, accepted in IEEE Communications Letters, 2013.

[9] ITU-R Recommendation P.618-10, Propagation data and prediction method required for the design of the Earth-space telecommunication systems, Geneva, 2009.

[10] Ko Young-Chai, M.-S. Alouini and Marvin K. Simon, Analysis and optimization of switched diversity systems, IEEE Transactions on Vehicular Technology, vol. 49, no. 5, pp. 1813-1831, 2000.

[11] H. A. David, Order Statistics, New York, NY: John Wiley \& Sons, Inc., 1981.

[12] ETSI EN 302307 v.1.1.2, Digital Video Broadcasting (DVB), Second generation framing structure, channel coding and modulation systems for broadcasting, interactive services, news gathering and other broadband satellite applications, 2003. 